\title{
Molecular mechanism of dengue infection and plant made vaccine treatment strategy: a review
}

\begin{abstract}
Dengue is the most common endemic disease in tropical region transmitted to humans by mosquitoes of Aedes species and often manifests to be fatal. In recent decades the prevalence and circulation of dengue has upturned dramatically. Unplanned development of cities without proper water management system is the main reason for spread of dengue. The true burden of dengue is unclear due to reported data. Dengue showing a wide range of symptoms is easily misdiagnosed and it does not have a specific treatment. With the increase in the number of dengue affected population there is an urge to develop effective and specific treatment for dengue. For unfolding this problem development of potential vaccine against dengue which is also cost effective would be an ideal fix. In this review we focus on using genetic engineering tools in turning plants as the biofactories for developing efficient vaccines against Dengue.
\end{abstract}

Keywords: dengue virus, aedes aegypti, vaccine development, plant molecular farming

\author{
Volume 7 Issue 5 - 2019
}

\author{
Harish MC, Susitra Priyadarshini M, Prakash \\ C \\ Department of Biotechnology, Thiruvalluvar University, India
}

Correspondence: Dr. Harish MC, Department of Biotechnology, Thiruvalluvar University, Serkkadu, Vellore 632 I I5, India, Tel +91 9842726952.

Emailmc.harishin@tvu.edu.in, mc.harishin@gmail.com

Received: September 21, 2019 | Published: October 31, 2019
Abbreviations: TBEV, tick borne encephalitis; YFV, yellow fever; WNV, west nile; JEV, Japanese encephalitis; prM, precursor membrane

\section{Introduction}

Dengue is one of the extensively spread mosquito-borne disease, transmitted by infected mosquitoes of Aedes species (Ae.aegypti, Ae.albopictus). ${ }^{1}$ Dengue fever is endemic in about 100 countries with majority of the cases reported from tropical regions of America, Pan Asia and Western pacific regions. The major factors for spread of dengue virus includes progression of virus, proliferation of climate dependent vectors, unplanned urbanization and increasing socioeconomic development. ${ }^{2}$ The virus is a single stranded RNA belonging to genus Flavivirus and family Flaviviridae, consisting of four serotypes DEN-1, DEN-2, DEN-3, and DEN-4 which causes dengue fever (DF). Every one of the serotypes is genetically related and antigenically distinct. ${ }^{3}$ The clinical implications of dengue consist of three phase which includes febrile, critical, and recovery phase. In most of the dengue cases the individuals are infected but show no symptoms. The viral genome encodes for three structural proteins viz capsid [C], envelope [E] and precursor membrane [prM]). The DENV E protein contributes in host cell attachment and viral entry and hence it is regarded as the major antigenic determinant. There are no specific treatments available for dengue until now. Recent development in plant biotechnology have paved away to employ plants as a potential system to produce vaccines. Plant production systems have advantages like, ease in growth and cultivation, lower contamination and production cost compared to mammalian culture systems and less downstream processing. Hence plants can be potentially used to raise vaccine against dengue virus.

\section{Epidemiology}

The prevalence of dengue in generally has increased approximately by 30 fold in last five decades. Dengue is considered to be endemic in almost $80 \%$ of the countries in Asia, South America and Africa and it is expected to be transmitted to tropical and subtropical regions where approximately 3.5 billion people lives. The World Health Organization (WHO) has predicted that the rise of infection can reach up to 100 million yearly, including 500,000 DHF cases and around 20,000 deaths, mostly children. ${ }^{4}$ During 1970's 9 nations had encountered extreme dengue pandemics; in present situation in excess of 100 nations have been influenced with dengue fever generally in tropical and subtropical locales. At current circumstance, dengue infection (DENV) represents a noteworthy danger to worldwide general wellbeing, and almost two-fifths of the total populace is in danger of dengue infection. ${ }^{5}$ Since last few decades the incidence of dengue infection has been dramatically increased in India. The first reported occurrence of dengue fever in India was in early 1960's in Kolkata. Almost after three decades a major dengue outbreak occurred in Delhi in 1996 reporting 10,000 positive cases. ${ }^{6}$ During this period DENV-2 were reported from all parts of India, signifying that this strain was predominantly circulating in India. Furthermore from mid of 2000's all four serotypes were reported from almost all the states in India. ${ }^{78}$ It is now evident that in India the frequency and number of outbreak has increased with respect to all four serotypes. It is now evident that in India the frequency and number if outbreak has increased with respect to all four serotypes. In Tamil Nadu dengue fever had been accounted for much of the time and being Chennai city as the real contributor. As of late, the general level of dengue cases in Chennai city declined to $45 \% .{ }^{9}$ High numbers of cases was recorded between September and December (North east storm season). All the four dengue serotypes had been reported in Chennai and other parts of Tamilnadu.

\section{Dengue virus structure}

Dengue virus is a member of the genus Flavivirus belonging to Flaviviridae family. The other important human pathogens of the same genus are: yellow fever (YFV), West Nile (WNV), Japanese encephalitis (JEV), and tick borne encephalitis (TBEV). The dengue virus is roughly spherical in shape..$^{10}$ The mature viron has structural 
proteins which includes the capsid $(\mathrm{C})$, the membrane $(\mathrm{M})$ and the envelope (E) proteins (Figure 1). The single standard RNA genome of the virus is surrounded by multiple copies of Capsid protein which forms the viral nucleocapsid (Figure 2). The $\mathrm{M}$ and $\mathrm{E}$ proteins are anchored in nucleocapsid surrounded by host cell derived lipid bilayer.

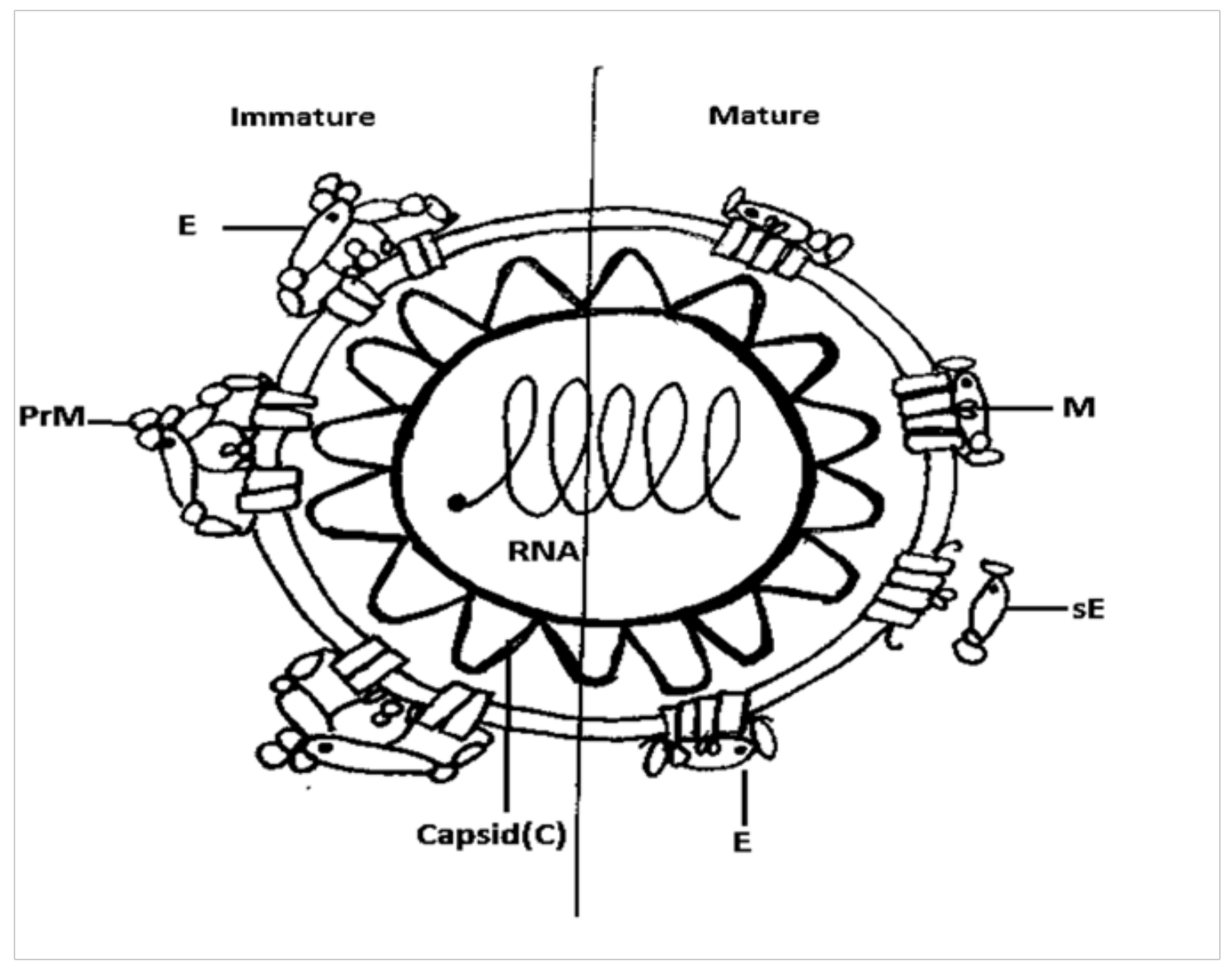

Figure I Schematic representation of a dengue virus particle. Left: immature virion; right: mature virion.

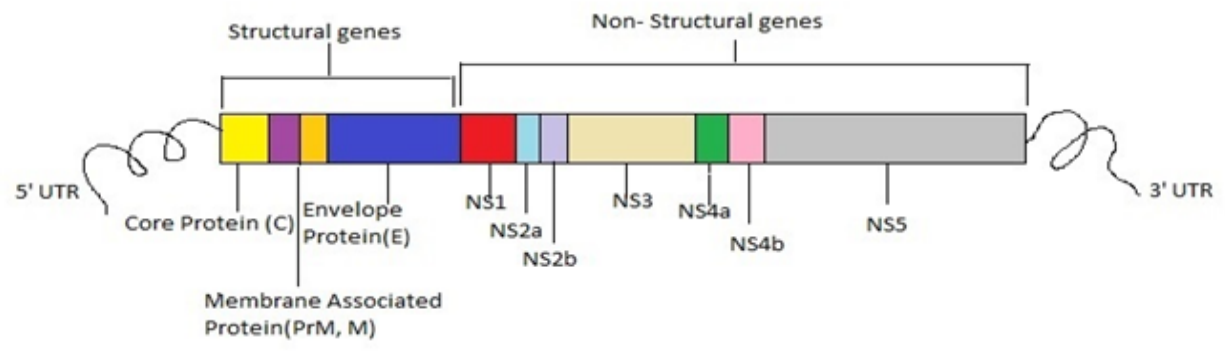

Figure 2 Dengue Virus genome showing 3 structural proteins genes (C, M and E) and 7 non-structural proteins (NSI, NS2a, NS2b, NS3, NS4a, NS4b and NS5. 


\section{Virus entry}

The virus enters into the host cells by receptor mediated endocytosis through cell surface receptors (Figure 3). The cellular glycoproteins receptors like heparin sulfates, dendritic cell-specific intercellular adhesion molecules and mannose are required for viral passage into the host cell. ${ }^{11,12}$ The viral E protein is responsible for host cell-receptor binding, viral entry, and hence elicits immunogenicity. The E protein is comprised of three domains: domain I (structural domain), domain II (dimers) harboring the fusion peptide at its distal tip, and domain III (receptor-binding). The mature E protein exists as a homodimer with the fusion peptide; the hydrophobic fusion peptide is exposed by low-pH induced trimerization in a manner consistent with membrane fusion mediated by class II fusion protein. ${ }^{13}$ Once inside the cell, the acidic $\mathrm{pH}$ in the endosome generates a conformational change in the E protein resulting in membrane fusion. The viral entry is mediated by the release of nucleocapsid into the cytoplasm of the host cell and then the virus un coats and releases the genome and positive-strand of viral RNA is translated into a single polyprotein, which is cleaved into the individual structural and NS proteins. ${ }^{14}$ After multiple rounds of translation high levels of viral proteins are produced that together with viral RNA are assembled into progeny virions. As a result of DENV infection vesicle packets (VPs) or smooth membrane structures (SMS) are formed in cytosol where the viral replication complex (RC) accumulates. $^{15}$

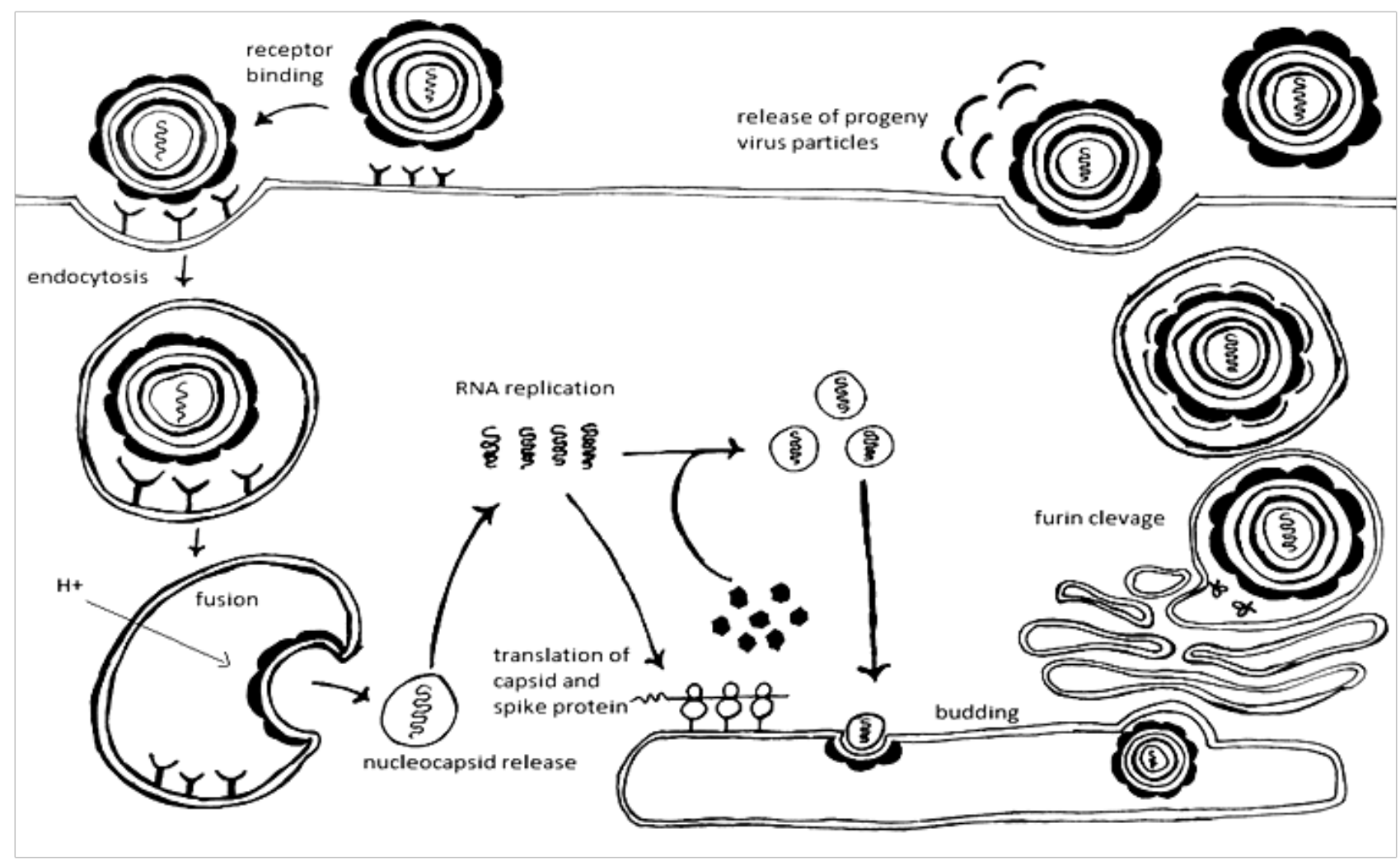

Figure 3 Life cycle of Dengue virus.

\section{Transmission cycles}

The principal vector $A$. aegypti causes dengue fever in humans by transmitting dengue virus through biting; the mosquito has small black-and-white bands on legs and body, highly domesticated in tropical region with cold climatic conditions. These mosquitoes are mostly associated with human living spaces and prefer to lay its eggs in fresh/rain water accumulated in containers, old automobile tires, broken pots, drums, buckets etc and generally spend life in and around human habitat. After hatching from eggs the larvae tend to live in aquatic environment where they feed on microorganisms, the fully grown larva will undergo the last stage called pupa and finally come out as adult mosquito. The male $A$. aegypti do not feed on human but the female mosquitoes are very nervous human feeders which make them to feed every 2 to 3 -hour interval.

\section{Symptoms}

Dengue being a multifarious disease with wide range of clinical symptoms is often misdiagnosed with other. The ailment ranges from asymptomatic infection, through undifferentiated fever and benign DF to severe hemorrhagic fever with or without shock syndrome. The incubation time frame ranges from 4-7 days yet now and then it stretches out to 14 days. Children often have less severe symptoms than adults and experience symptoms similar to those of the common cold and gastroenteritis, but are more susceptible to the severe complications. Symptoms may include, high fever, headaches, vomiting, skin rash, severe joint, muscle pain and mild bleeding from nose, gums etc., which appears 2 to 5 days after the onset of fever. ${ }^{16}$ 


\section{Treatment}

On account of non-availability of specific anti- dengue drug the treatment for dengue is supportive rather than being curative, and treatment mainly depends upon management of fluid intake (either oral or intravenous) to avoid circulatory shock. The only way to control dengue epidemic is vector control. The spread of dengue in the last two decades have increased due to the lack of specified antiviral medications and vaccine, and difficulties in controlling the major global vector Aedes aegypti mosquitoes. Hence the disease could be managed by careful attention of fluid management and proactive treatment of hemorrhage.

\section{Vaccine development}

Previous research had paved a way to develop vaccine such as, chimeric virus, live attenuated virus, recombinant subunit vaccines, DNA vaccines and vector based vaccines. Among the above mentioned vaccines development of dengue subunit vaccine is also gaining popularity among researchers; it has both advantages as well as disadvantage over live attenuated vaccine. The major advantage of a subunit vaccine for dengue is that it would be a lot easier to balance the level and immunogenicity of the four component parts to provide complete protection against all four serotypes. Therefore, the production cost of the subunits could be dramatically reduced. Subunit vaccines require large amounts of purified antigen to be used in the vaccine development, as well as the use of adjuvants, both serve to increase the cost of the vaccine. The mammalian cellculture systems used to produce dengue antigens tend to have low yields of the antigen, coupled with the potential of contamination with unknown pathogenic agents.

Owing to the disadvantages in other cell culture systems, plants can be used as biofactories for production of vaccines in large quantities i.e. by plant molecular farming, in which plants are genetically modified to produce desired protein of interest. In this case plants can be genetically modified using various molecular biology tools to express subunit vaccine for dengue. Plant cells can accumulate foreign proteins in cell organelles without disturbing its native biological activity of the protein. Hence, plant based vaccine production system also reduces the cost of down streaming process, thus it is cost effective. Several plant derived vaccines for human disease are in clinical trial. ${ }^{[44]}$ Plant based vaccines are proven to be cheap and they are also easy to scale up. With the emergence of plant molecular farming many research groups working globally in development vaccines against viral diseases through plants. Recently plant based vaccines against Hepatitis B virus, influenza virus, poliovirus, papillomavirus, human immunodeficiency virus were developed and few of them are in clinical trials. ${ }^{17}$ Regardless of success in currently available vaccines against the above mentioned diseases, there is still scope for development of safe, cheap and efficient vaccines. The first such plant made edible vaccine was produced in potato for hepatitis B by expressing S-HBsAg and later in lettuce plants which was further formulated for oral delivery. ${ }^{18}$ Recently virus like particle (VLP) was produced in Nicotiana benthamiana against polio virus and the expression of recombinant Polio Virus which yielded up to $60 \mathrm{mg} / \mathrm{kg}$ in infiltrated plant tissue. ${ }^{19}$ Another study demonstrated the improved immunogenicity against papillomavirus when the HPV-16L1 protein (C terminal protein) was fused with heat labile enterotoxin B (LTB) and expressed in chloroplast of tobacco plant by direct coupling of an antigen with an adjuvant. ${ }^{20}$ Currently the Plant based HIV neutralizing antibodies are gaining more interest which is consider to have both prophylactic and therapeutic use and hence some of these broadly neutralizing anti-HIV monoclonal antibodies like 2G12, (a high mannose cluster in the $\mathrm{C} 3-\mathrm{V} 4$ region); a single chain $\mathrm{m} 9$, (CD4inducible) and VRC01 (CD4 bs) were transiently and expressed in Nicotiana tabaccum and Nicotiana benthamiana plants and the plant derived $\mathrm{mAbs}$ showed similar activity to the counterparts produced in CHO and HEK-293 cells. ${ }^{21}$ These studies demonstrate the possibilities of plants as an efficient platform for recombinant vaccines.

In this decade much attention have attained towards the production vaccine in plants for vector borne diseases and particularly viral disease of genus Flavivirus (Table 1). ${ }^{22-30}$ Majority of these studies focuses on domain III of envelope protein (EDIII) as a potential vaccine candidate against Flavivirus (Zika, Yellow fever and Dengue). ${ }^{25}$ Hence our group is also focusing on expression of EDIII protein of dengue virus in plants, which would acts as vaccine candidate against dengue virus. Earlier development of dengue virus vaccine was based on YFV (Yellow Fever Virus) which has structural similarities with dengue virus and both belong to the same genus Flaviviridae. The first developed vaccine for YFV was a live attenuated vaccine called French neurotrophic vaccine. ${ }^{24}$ Hence the efforts to develop vaccine against dengue are focused on development of live attenuated vaccines. Development of an effective vaccine against dengue virus remains as a challenge due to the different serotypes that all are lethal to humans. The monovalent vaccines have shown some promise where as in tetravalent vaccines there are difficulties in balancing the four serotypes and to provide uniform protection. In this manner, the perfect vaccine must be immunogenic against each of the four serotypes as opposed to one. It has been demonstrated that protection with one serotype will offer long haul security against that serotype, yet presumably just momentary assurance against the others. Thus, for those getting a second infection with an alternate serotype, the seriousness might be more noteworthy. One more hurdle in development of dengue vaccine is antibody dependent enhancement (ADE), a phenomenon in which every serotype provides lifelong immunity but infection with heterologous virus enhances the disease severity. This condition is quite common in children born to dengueimmune mothers. ${ }^{22}$ Therefore, a hypothetical risk exists in developing a monovalent immunization in light of the fact that regardless of whether it is effective in giving invulnerability against one serotype, the risk might be more for recipients whenever tainted with an alternate serotype. So the current endeavors are generally coordinated towards building up a monovalent vaccine.

Hence, the envelope E protein of dengue virus has gained attention on the development of recombinant DNA based vaccine, because the first step of DENV infection is to attach with host through host receptor and the envelope protein $\mathrm{E}$ is majorly involved in the attachment of virus and host, hence targeting the envelope $\mathrm{E}$ protein for vaccine development would be ideal for inhibiting the host-viral interaction. ${ }^{25,26}$ The E glycoprotein (495aa) is the major structural protein exposed on the surface of mature dengue virus and also plays an important role in host cell attachment and entry. The E glycoprotein consists of three structurally distinct domains- I, II and III. The dengue E glycoprotein is effectively produced by several bacterial culture systems. It is shown in previous studies that the expression level of 
full length or ectodomain of DEN 2E protein is low when produced in other expression system such as mammalian cell culture or bacterial culture and tends to degrade easily. The existing culture system also failed to exhibit antibody neutralizing activity against DEN-2. Gene encoding domain 3 of dengue 2 envelope protein (D2EIII) was successfully expressed in model plant Nicotina benthamiana using tobacco mosaic virus based transient expression. ${ }^{19}$ Antibody against dengue was produced when mouse is immunized with D2EIII and also established neutralizing activity against DEN-2.

Table I List of plant based vaccines for Flavivirus

\begin{tabular}{llll}
\hline Virus & Expressed antigen & Host plant & Reference \\
\hline Zika & HBcAgzEDIII VLP & Nicotinabenthamiana & {$[23]$} \\
Yellow & YFE & Nicotinabenthamiana & {$[24]$} \\
Fever & & & \\
& I) Consensus & & \\
& EDIII(cEDIII) & Nicotinabenthamiana & {$[25]$} \\
Dengue & 2) cEDIII fusion to & & \\
& M-cell targeting ligand & & \\
& (Col) & & {$[26]$} \\
& Tetravalent fusion of & Nicotinatabacum & [27] \\
Dengue & EDIII & Oryza sativa & {$[28]$} \\
Dengue & cEDIII-Col & Oryza sativa & {$[29]$} \\
Dengue & DENV2- specific E & & \\
& DENV2- specific & Zea mays & \\
Dengue & I) EDIII & & \\
& 2) EDIII-CTB & Curcurbitapepo & \\
\hline Dengue & DENV2- specific EDIII & &
\end{tabular}

\section{Conclusion}

Due to the non-availability of specific treatment, dengue infection has been a serious issue globally starting from its first outbreak. Vector control was the only preventive option to keep the viral spread in control. There is an urgent need for vaccine against dengue virus to manage its infection. Dengue virus envelope protein E plays a major role in virus entry; hence it can serve as an important drug target to develop vaccine against dengue virus in a plant based system.

\section{Funding}

None.

\section{Acknowledgements}

None.

\section{Conflict of interest}

The author declares that there is no conflict of interest.

\section{References}

1. Atique S, Abdul SS, Hsu CY, et al. Meteorological influences on dengue transmission in Pakistan. Asian Pacific Journal of Tropical Medicine. 2016;9(10):954-961.

2. Rafique I, Saqib MAN, Munir MA, et al. Asymptomatic dengue infection in adults of major cities of Pakistan. Asian Pacific Journal of Tropical Medicine. 2017;10(10):1002-1006.
3. Pang EL, Loh HS. Towards development of a universal dengue vaccine-How close are we? Asian Pacific Journal of Tropical Medicine. 2017;10(3):220-228.

4. Guo C, Zhou Z, Wen Z, et al. Global Epidemiology of Dengue Outbreaks in 1990-2015: A Systematic Review and Meta-Analysis. Frontiers in Cellar Infection Microbiology. 2017;7:1-317.

5. Anne Murray NE, Quam MB, Wilder-Smith A. Epidemiology of dengue: past, present and future prospects. Clinical Epidemiology. 2013;5:299309.

6. Chaturvedi UC, Kapoor AK, Mathur A, et al. A clinical and epidemiological study of an epidemic of febrile illness with haemorrhagic manifestations which occurred at Kanpur, India in 1968. Bulletin of World Health Organisation. 1970;43:281-287.

7. Chaturvedi UC, Mathur A, Kapoor AK, et al. Clinicovirological study of the recurrence of dengue epidemic with haemorrhagic manifestation at Kanpur, during 1969. Indian Journal of Medical Research. 1972;60:329333 .

8. Chaturvedi UC, Mathur A, Mehrotra RML. Experimentally produced cardiac injury following dengue virus infection. Indian Journal of Pathology and Bacteriology. 1974;17:218-220.

9. Mutheneni SR, Morse AP, Caminade C, et al. Dengue burden in India: recent trends and importance of climatic parameters. Emerging Microbes and Infections. 2017;6(8):e70.

10. Kuhn RJ, Zhang W, Rossmann MG, et al. Structure of dengue virus: implications for flavivirus organization, maturation, and fusion. Cell. 2002;108:717-725.

11. Modis Y, Ogata S, Clements D, et al. Variable surface epitopes in the crystal structure of dengue virus type 3envelope glycoprotein. Journal of Virology. 2005;79:1223-1231.

12. Bryant JE, Calvert AE, Mesesan K, et al. Glycosylation of the dengue 2 virus $\mathrm{E}$ protein at $\mathrm{N} 67$ iscritical for virus growth in vitro but not for growth in intra-thoracically inoculated Aedes aegypti mosquitoes. Virology. 2007;366:415-423.

13. Crabtree MB, Kinney RM, Miller BR. Deglycosylation ofthe NS1 protein of dengue 2 virus, strain 16681: constructionand characterization of mutant viruses. Archives of Virology. 2005;150:771-786.

14. Zhang Y, Corver J, Chipman PR, et al. Structures of immature flavivirus particles. EMBO Journal. 2003;22:2604-2613.

15. Hunt AR, Cropp CB, Chang GJ. A recombinant particulate antigen of Japanese encephalitis virus produced in stably transformed cells is an effective noninfectious antigen and subunit immunogen. Journal of Virological Methods. 2001;97:133-149.

16. Chawla P, Yadav A, Chawla V. Clinical implications and treatment of dengue. Asian Pacific Journal of Tropical Medicine. 2014;7(3):169-178.

17. Rybicki EP. Plant-based vaccines against viruses. Virology Jornal. 2014;11:205.

18. Marsian J, Fox H, Bahar MW, et al. Plant-made polio type 3 stabilized VLPs-a candidate synthetic polio vaccine. Nature Communications. 2017;8(1):245.

19. Pniewski T, Kapusta J, Bociag P, et al. Low-dose oral immunization with lyophilized tissue of herbicide-resistant lettuce expressing hepatitis B surface antigen for prototype plant-derived vaccine tablet formulation. Journal of Applied Genetics. 2011;52:125-136.

20. Whitehead M, Ohlschlager P, Almajhdi FN, et al. Human papillomavirus (HPV) type 16 E7 protein bodies cause tumour regression in mice. $B M C$ Cancer. 2014;14:367. 
21. Rosenberg Y, Sack M, Montefiori D, et al. Rapid high-level production of functional HIV broadly neutralizing monoclonal antibodies in transient plant expression systems. PLoS One. 2013;8:e58724

22. Thomas SJ, Endy TP. Critical issues in dengue vaccine development. Current Opinion in Infectious Diseases. 2011;24:442-450.

23. Yang M, Lai H, Sun H, et al. Virus-like particles that display Zika virus envelope protein domain III induce potent neutralizing immune responses in mice. Sci Rep. 2017;7(1):7679

24. Tottey S, Shoji Y, Jones RM, et al. Plant-Produced Subunit Vaccine Candidates against Yellow Fever Induce Virus Neutralizing Antibodies and Confer Protection against Viral Challenge in Animal Models. American Journal of Tropical Medicine and Hygiene. 2017;98(2):420-431.

25. Kim MY, Jang YS, Yang MS, et al. High expression of consensus dengue virus envelope glycoprotein domain III using a viral expression system in tobacco. Plant Cell Tissue and Organ Culture. 2015;122:445-451.

26. Gottschamel J, Lössl A, Ruf S, et al. Production of dengue virus envelope protein domain III-based antigens in tobacco chloroplasts using inducible and constitutive expression systems. Plant Molecular Biology. 2016;91:497-512.
27. Kim TG, Kim MY, Huy NX, et al. M cell-targeting ligand and consensus dengue virus envelope protein domain III fusion protein production in transgenic rice calli. Molecular Biotechnology. 2013;54:880-887.

28. Kim TG, Kim MY, Tien NQD, et al. Dengue virus E glycoprotein production in transgenic rice callus. Molecular Biotechnology. 2014;56:1069-1078.

29. Kim HA, Kwon SY, Yang MS, et al. Expression of Dengue virus EIII domain-coding gene in maize as an edible vaccine candidate. Journal of Plant Biotechnology. 2014;41:50.

30. Libsittikul S, Khongwichit S, Smith DR, et al. Evaluation of papaya ringspot virus as a vector for expression of dengue E protein domain III in Cucurbitapepo (Zucchini) plants. Journal of Animal and Plant Sciences. 2015;25(3):809-815. 